\title{
Post-warm-up muscle temperature maintenance: blood flow contribution and external heating optimisation
}

\author{
Margherita Raccuglia ${ }^{1,2}$ • Alex Lloyd ${ }^{1}$ - Davide Filingeri ${ }^{1}$ - Steve H. Faulkner ${ }^{3}$. \\ Simon Hodder ${ }^{1}$ George Havenith ${ }^{1}$
}

Received: 5 August 2015 / Accepted: 4 November 2015 / Published online: 21 November 2015

(c) The Author(s) 2015. This article is published with open access at Springerlink.com

\begin{abstract}
Purpose Passive muscle heating has been shown to reduce the drop in post-warm-up muscle temperature $\left(T_{\mathrm{m}}\right)$ by about $25 \%$ over $30 \mathrm{~min}$, with concomitant sprint/power performance improvements. We sought to determine the role of leg blood flow in this cooling and whether optimising the heating procedure would further benefit post-warmup $T_{\mathrm{m}}$ maintenance.

Methods Ten male cyclists completed 15-min sprintbased warm-up followed by $30 \mathrm{~min}$ recovery. Vastus lateralis $T_{\mathrm{m}}\left(T_{\mathrm{mvl}}\right)$ was measured at deep-, mid- and superficial-depths before and after the warm-up, and after the recovery period (POST-REC). During the recovery period, participants wore water-perfused trousers heated to $43{ }^{\circ} \mathrm{C}$ (WPT43) with either whole leg heating (WHOLE) or upper leg heating (UPPER), which was compared to heating with electrically heated trousers at $40{ }^{\circ} \mathrm{C}$ (ELEC40) and a nonheated control $(\mathrm{CON})$. The blood flow cooling effect on $T_{\text {mvl }}$ was studied comparing one leg with (BF) and without (NBF) blood flow.

Results Warm-up exercise significantly increased $T_{\text {mvl }}$ by $\sim 3{ }^{\circ} \mathrm{C}$ at all depths. After the recovery period,

Communicated by Narihiko Kondo.

George Havenith

G.Havenith@lboro.ac.uk

1 Environmental Ergonomics Research Centre, Loughborough Design School, Loughborough University, Loughborough, Leicestershire LE11 3TU, UK

2 Sport and Exercise Sciences Research Unit, University of Palermo, Palermo, Italy

3 School of Sport, Exercise and Health Sciences, Loughborough University, Loughborough, Leicestershire LE11 3TU, UK
\end{abstract}

BF $T_{\text {mvl }}$ was lower $\left(\sim 0.3{ }^{\circ} \mathrm{C}\right)$ than NBF $T_{\text {mvl }}$ at all measured depths, with no difference between WHOLE versus UPPER. WPT43 reduced the post-warm-up drop in deep- $T_{\text {mvl }}\left(-0.12{ }^{\circ} \mathrm{C} \pm 0.3{ }^{\circ} \mathrm{C}\right)$ compared to ELEC40 $\left(-1.08 \pm 0.4{ }^{\circ} \mathrm{C}\right)$ and $\mathrm{CON}\left(-1.3 \pm 0.3{ }^{\circ} \mathrm{C}\right)$, whereas mid- and superficial- $T_{\text {mvl }}$ even increased by $0.15 \pm 0.3$ and $1.1 \pm 1.1^{\circ} \mathrm{C}$, respectively.

Conclusion Thigh blood flow contributes to the postwarm-up $T_{\mathrm{mvl}}$ decline. Optimising the external heating procedure and increasing heating temperature of only $3{ }^{\circ} \mathrm{C}$ successfully maintained and even increased $T_{\text {mvl }}$, demonstrating that heating temperature is the major determinant of post-warm-up $T_{\mathrm{mvl}}$ cooling in this application.

Keywords Muscle temperature $\cdot$ Blood flow $\cdot$ Passive heating $\cdot$ Water perfused trousers $\cdot$ Occlusion

$\begin{array}{ll}\text { Abbreviations } \\ \text { BF } & \text { Blood flow } \\ \text { CON } & \text { Control (non-heated) } \\ \text { ELEC40 } & \text { Electrically heated trousers to } 40{ }^{\circ} \mathrm{C} \\ \text { NBF } & \text { No blood flow } \\ T_{\text {c }} & \text { Core temperature } \\ T_{\text {mvl }} & \text { Vastus lateralis muscle temperature } \\ T_{\text {sk-gas }} & \text { Gastrocnemius skin temperature } \\ T_{\text {sk-quad }} & \text { Quadriceps skin temperature } \\ \text { UPPER } & \text { Upper leg heating } \\ \text { WHOLE } & \text { Whole leg heating } \\ \text { WPT43 } & \text { Water perfused trousers heated to } 43{ }^{\circ} \mathrm{C}\end{array}$

\section{Introduction}

The importance of warming-up for subsequent shortduration/power-based exercise performance has been well 
documented (Asmussen and Boje 1945; Bergh and Ekblom 1979; Sargeant 1987; Hajoglou et al. 2005). Although prior exercise may induce psychological (Malareki 1954) and neuromuscular changes (Bishop 2003a, b) with a beneficial effect on performance, it has been suggested that the major contributing factor to post-warm-up performance improvement is the rise in muscle temperature $\left(T_{\mathrm{m}}\right)$ (Bishop 2003a, b). Generally, $T_{\mathrm{m}}$ increases rapidly within the first $3-5 \mathrm{~min}$ of exercise, reaches a plateau after 10-20 min of activity and drops exponentially within 15-30 min after cessation of exercise (Saltin 1968; Faulkner et al. 2013b). It has been indicated that a recovery time of 15-20 min, between warm-up completion and the start of a sport event, allows for acid-base homeostasis (Bishop 2001), optimal balance between phosphocreatine restoration (Dawson et al. 1997) and muscle potentiation (Kilduff et al. 2013). However, it is not uncommon for athletes to experience a significantly longer recovery period (30-45 min) between active warmup completion and their subsequent exercise performance (Mohr et al. 2004; Kilduff et al. 2013; West et al. 2013). For instance, cyclists perform a cycle-based warm-up approximately $30 \mathrm{~min}$ before the race, and this long delay has been shown to cause a significant reduction in $T_{\mathrm{m}}$, which has a detrimental effect on the following sprint performance (Faulkner et al. 2013a, b).

Our two previous studies have demonstrated that the use of heated trousers, during this period of inactivity, results in a significant attenuation in the vastus lateralis $T_{\mathrm{m}}\left(T_{\mathrm{mvl}}\right)$ drop (Faulkner et al. 2013a, b). The attenuated drop in $\mathrm{T}_{\text {mvl }}$ was consistently associated with a greater peak and mean power output ( $\sim-11$ and $\sim 4 \%$, respectively) during 30-s maximal sprint, confirming the beneficial effect of an increased starting $T_{\mathrm{m}}$ on power-based performance. Nevertheless, despite the effectiveness of the heated trousers, a significant $T_{\text {mvl }}$ drop of over $1.5^{\circ} \mathrm{C}$ was still observed over the course of the recovery period. It has been suggested that leg blood flow could be a potential contributing factor to the decline in post-warm-up $T_{\text {mvl }}$ (Faulkner et al. 2013a, b). In this regard, Kenny et al. (2003) suggested that postexercise core temperature response is significantly influenced by conductive heat transfer from muscle to venous blood with subsequent convective transfer by the blood to the body core. In support Ducharme and Tikuisis (1994) showed that during immersion of the forearm and hand in water at $20{ }^{\circ} \mathrm{C}$, heat exchange through convection between the blood and the forearm tissues accounted for $85 \%$ of the total heat transferred. Conversely, during water immersion at $38{ }^{\circ} \mathrm{C}$, the blood has the role of heat sink, transferring heat gained by the tissues from the environment to the rest of the body (Ducharme and Tikuisis 1994). According to the heat transfer analysis (Havenith 2001), the lower temperature of central blood, flowing into the warmer leg muscle tissue (post-warm-up), could have caused the previously observed reduction in $T_{\text {mvl }}$, despite external heating. Furthermore, contributing to this may have been the effect of cooled blood returning from the lower leg and foot, subsequently cooling the muscle of the thigh as it returns to the body core. However to date, no research has examined the role of blood flow on $T_{\mathrm{mvl}}$ reduction, while using passive heating.

Another contributor to our previously observed reduction in $T_{\text {mvl }}$ could be the microclimate created between the skin and trousers. It is possible that the previously adopted heating method did not provide enough heat to maintain $T_{\text {mvl }}$. In fact, for safety reasons, the heating elements of the previously used heating trousers were designed not to exceed a temperature of $40{ }^{\circ} \mathrm{C}$. Using electrical heating (Faulkner et al. 2013a, b), a safety margin for the heater temperature is required to avoid skin burns $\left(45^{\circ} \mathrm{C}\right)$ as changes to the heating element insulation (e.g. sitting on a chair) will change the temperature achieved by the heaters.

In order to investigate the aforementioned mechanisms (i.e. blood flow and optimal microclimate temperature), in the current study lower limb arterial and venous blood circulation was restricted in a single leg during the postwarm-up recovery. As such, the contribution of blood flow, as a cooling source, was studied by comparing $T_{\text {mvl }}$ between the occluded and the perfused leg. Furthermore, to investigate the role of cooled blood returning to the thigh from the unheated lower leg (Faulkner et al. 2013a, b) (via venous return), post-recovery $T_{\mathrm{mvl}}$ was observed in two different conditions: whole leg heated (WHOLE) and upper leg heated only (UPPER).

Lastly, using circulating liquid heating, local overheating (encountered with the electrically heated trousers) does not occur; therefore, the application of a liquid heating system allowed us to increase the external temperature from $\sim 40$ to $43{ }^{\circ} \mathrm{C}$ (without any skin damage), and thus, the study of the role of an increased heating temperature (microclimate) on $T_{\mathrm{mvl}}$ declines.

The occlusion of the leg blood flow, required to answer the research question posed, causes muscle ischemia and a numb leg and therefore precludes this experimental design from incorporating a performance test. The impact of $T_{\mathrm{m}}$ on performance has been repeatedly demonstrated (Asmussen and Boje 1945; Bergh and Ekblom 1979; Sargeant 1987; Hajoglou et al. 2005; Faulkner et al. 2013a, b), and a positive dose-response relation for post-warm-up $T_{\mathrm{m}}$ and performance is assumed (more detail on this will be presented in discussion). Therefore, a performance test was not considered necessary to answer the research question posed.

In summary, the purpose of this study was twofold. First, we aimed to investigate the role of central (core) and peripheral (lower leg) blood flow on $T_{\mathrm{mvl}}$ during $30 \mathrm{~min}$ of passive recovery. We hypothesised that $T_{\mathrm{mvl}}$ would be (1) lower in the perfused leg compared to the occluded leg, due 
to the heat loss to arterial blood coming from the core and (2) lower in the upper leg heating only versus whole leg heating, due to the higher heat loss to the cooled venous blood returning from the unheated lower leg and foot. Secondly, we aimed to examine the effect of heating temperature on $T_{\mathrm{mvl}}$ during the course of the passive recovery period. We hypothesised that the application of an optimised (higher) heating temperature will further reduce the previously observed drop in $T_{\mathrm{mvl}}$ over the course of the recovery period. To assess the latter, $T_{\mathrm{mvl}}$ results achieved by water-perfused trousers $\left(43{ }^{\circ} \mathrm{C}\right.$ heating temperature) were compared to $T_{\mathrm{mvl}}$ data obtained from our previous heating method (up to $40^{\circ} \mathrm{C}$ ) and control group (no passive heating), in which the exercise protocol adopted was identical to that of the current study.

\section{Methods}

\section{Participants}

Ten male cyclists volunteered to participate in this investigation (age $22.0 \pm 0.8$ years; height $180.0 \pm 2.3 \mathrm{~cm}$; body mass $76.5 \pm 5.4 \mathrm{~kg}$; mean $\pm \mathrm{SD}$ ). With respect to the training status of the participants, all of them performed at least three cycle-based training sessions per week as well as other regular physical activity, such as running and weight lifting. They completed a general health-screening questionnaire and were all non-smokers, free from injury and from any medication. All subjects were informed verbally of the objectives and procedures of the study before giving written informed consent. In the $24 \mathrm{~h}$ prior to each trial, participants were asked to refrain from caffeine, alcohol ingestion and any strenuous exercise. They were also asked to keep a record of their food intake and replicate this prior to the subsequent visit. The protocol and procedures involved were approved by the Loughborough University Ethical Advisory Committee. The study was conducted within the confines of the World Medical Association Declaration of Helsinki for medical research using human participants.

For the comparison of different heating temperatures/ methods, a between-group comparison was made to the data presented by Faulkner et al. (2013a, b). Eleven male cyclists (age $=24.7 \pm 4.2$ years, height $=1.82 \pm 0.72 \mathrm{~m}$, body mass $=77.9 \pm 9.8 \mathrm{~kg}$; not significantly different from the present experimental group) with the same level of weekly activity were exposed to the exact same warm-up and recovery protocol.

\section{Study overview}

Participants visited the laboratory on three occasions. Prior to the main experimental trials, participants were familiarised with exercise testing and the general measurement procedures used in the present study. On the two remaining visits, participants performed the experimental tests. On each occasion, after $30 \mathrm{~min}$ of stabilisation period, participants completed a 15 -min cycle sprint warm-up, followed by $30 \mathrm{~min}$ of passive recovery. In both experimental trials, during the passive recovery, arterial and venous blood flow of one leg was occluded (NBF), whereas the other leg was used to observe the effect of blood flow on thigh muscle temperature decline (BF). The two visits differed in the surface area over which heat was applied; in one visit, upper and lower leg were warmed up (WHOLE), while in the other visit only the upper leg was kept warm (UPPER). The trials were completed in a balanced order, separated by a minimum of $72 \mathrm{~h}$. In order to make the current results comparable to that from previous studies (Faulkner et al. 2013a, b), all experiments were performed in a temperature and humidity-controlled environment maintained at $17.7 \pm 0.3{ }^{\circ} \mathrm{C}$ and $54.0 \pm 3.0 \%$ relative humidity, which Faulkner et al. (2013a, b) considered a range relevant to athletic events in a Western European climates (originally aiming at the London Olympics).

\section{Experimental protocol}

On the two experimental sessions, participants entered the controlled climatic room environment and were instrumented with skin, rectal and heart rate measurements systems. Participants remained lying on a bench for $30 \mathrm{~min}$, wearing shorts and t-shirt, to allow time for muscle, core and skin temperatures to stabilise. After the stabilisation period, baseline measures for thigh muscle temperature $\left(T_{\mathrm{mvl}}\right)$ at three different depths for both legs, quadriceps skin temperature ( $\left.T_{\text {sk-quad }}\right)$, gastrocnemius skin temperature $\left(T_{\text {sk-gas }}\right)$, core temperature $\left(T_{\mathrm{c}}\right)$ and heart rate $(\mathrm{HR})$ were taken. These parameters were recorded every $1 \mathrm{~min}$ during the experimental trials, apart from $T_{\mathrm{mvl}}$, which was recorded after the stabilisation period (PRE), on completion of the warm-up exercise (POST-WUP) and immediately after the recovery period (POST-REC). After the stabilisation period, participants mounted a cycle ergometer and performed the standardised active warm-up protocol which consisted of 5-min cycling at an external power output of $100 \mathrm{~W}$, followed by five 10-s maximal sprints (10-s Wingate test), each sprint separated by 1 min $55 \mathrm{~s}$ of cycling at $75 \mathrm{~W}$ (Faulkner et al. 2013a, b). Participants were asked to maintain a cadence of $85 \mathrm{rpm}$, until the start of each sprint. The maximal sprints were performed at a frictional load equivalent to $10 \%$ body mass. On completion of the warm-up, participants dismounted the ergometer, laid supine on the bench (this took $\sim 30 \mathrm{~s}$ ), where POST-WUP $T_{\mathrm{mvl}}$ was measured. Following this, a blood pressure cuff was immediately applied proximally around 
the right thigh to abolish/minimise leg blood flow, and the participant donned the water-perfused trousers (WPT). The mean inflation pressure was $140 \mathrm{mmHg}$. To ensure that the inflation pressure was supra-systolic, individual blood pressure was measured before the recovery period. During all conditions, the WPT were connected to a controlled water bath $\left(45.0 \pm 0.1{ }^{\circ} \mathrm{C}\right)$ and reached a temperature of $43.0 \pm 0.5{ }^{\circ} \mathrm{C}$. This temperature was selected after extensive pilot testing, as it was the maximum temperature for which no signs of superficial skin injury or pain were present. Once donned, the participants remained in the environmental chamber in a supine position for $30 \mathrm{~min}$. At the end of the 30-min recovery, the water-perfused trousers were removed and a POST-REC $T_{\mathrm{mvl}}$ measurement was taken. After this measurement, the blood pressure cuff was removed and the participant remained in the lying position until the stabilisation reached baseline parameters.

In addition, to assess the effectiveness of the optimised heating procedure (WPT43) in maintaining postwarm-up $T_{\text {mvl }}$, POST-REC $T_{\text {mvl }}$ data were compared with $T_{\mathrm{mvl}}$ data from two of our previous studies, where participants donned electrically heated trousers heated at $40{ }^{\circ} \mathrm{C}$ (ELEC40) and non-heated tracksuit bottoms (CON) during 30 min of passive recovery subsequent to the same warmup exercise protocol used in this study. The current group of subjects (WPT43) and those from our previous studies (ELEC40 and CON) were homogenous in terms of gender, age, anthropometric characteristics and fitness level (Faulkner et al. 2013a, b).

\section{Measurements}

\section{Core and skin temperature}

To monitor changes in core temperature, rectal temperature $\left(T_{\mathrm{c}}\right)$ was recorded throughout the experimentation. Participants inserted a rectal thermistor (Grant Instrument Ltd, Cambridge, UK) $10 \mathrm{~cm}$ beyond the anal sphincter. Local skin temperature over both quadriceps $\left(T_{\text {sk-quad }}\right)$ and gastrocnemius $\left(T_{\text {sk-gas }}\right)$ was measured using calibrated thermistors (Grant Instrument Ltd, Cambridge, UK), two for each region, placed at the following sites on the legs: muscle belly of rectus femoris and the widest diameter of the gastrocnemius. Temperatures were recorded at 1-min intervals using a Grant Squirrel 2010 data logger (Grant Instrument Ltd, Cambridge, UK).

\section{Muscle temperature}

Muscle temperature of the vastus lateralis of each leg was measured using a solid needle probe with an inbuilt thermocouple (Ellab, Copenhagen, Denmark). The following muscle temperature measurement procedure was developed after extensive pilot studies. The needle was inserted $1 / 3$ distal to the line that runs from the insertion of the vastus lateralis to the iliac crest. The insertion of the vastus lateralis was estimated at $1 / 4$ distal of the distance between the midpoint of the base of patella and the midpoint of popliteal fossa.

The insertion depth of the needle probe was corrected for adipose tissue. The adipose tissue thickness was calculated using a skinfold calliper over the site of the insertion. The average skinfold thickness of participants was $6 \pm 0.8 \mathrm{~mm}$.

The needle was inserted at 25,15 and $5 \mathrm{~mm}$ depths beyond the muscle fascia according to

Depth of insertion $=$ required depth $(\mathrm{mm})$

$+\frac{1}{2}$ local skinfold thickness $(\mathrm{mm})$.

Thus, in this experiment, the average depth of insertion (including adipose calculation) was $28 \mathrm{~mm}$ (deep- $\left.T_{\mathrm{mvl}}\right)$, $18 \mathrm{~mm}$ (mid- $T_{\mathrm{mvl}}$ ) and $8 \mathrm{~mm}$ (superficial- $T_{\mathrm{mvl}}$ ). To measure $T_{\mathrm{mvl}}$ at each depth, the needle probe was inserted at the deepest depth of $28 \mathrm{~mm}$, then withdrawn to $18 \mathrm{~mm}$, and finally withdrawn again to $8 \mathrm{~mm}$ depth. The needle was inserted at the same location for each measurement period (PRE, POST-WUP and POST-REC). To allow for stabilisation of the measurement, the needle was inserted for $\sim 3 \mathrm{~s}$ at each depth. The temperature was measured using a handheld digital thermometer (Ellab, Copenhagen, Denmark).

\section{Heart rate}

Heart rate was recorded every 5 min using a wireless heart rate monitor (RS800, Polar, Kempele, Finland).

\section{Water-perfused trousers}

During the 30-min recovery period, muscle passive heating was achieved using bespoke high-density water-perfused trousers (WPT; Med-Eng System Inc., Pembroke, Canada) consisting of 2.5 -mm-internal diameter medical grade PVC tubing, sewn in a pair of trousers. The tubing covered the following individually perfused segments: buttocks, front and back upper legs, and lower legs. The WPT were composed of spandex mesh fabric. The garment has a series of snap fasteners on each side running from the groin region to the ankles to ensure a tight fit. Additionally, to allow an adjustment of the tightness, two strips were integrated around each leg, as well as to minimise heat transfer between the hot water running through the suit and the cooler air temperature of the environmental chamber, a cover garment was placed over the WPT. The water flow was always kept at a fixed value of $250 \mathrm{~cm}^{3} /$ $\min$ (total $1000 \mathrm{~cm}^{3} / \mathrm{min}$ ) for each of the four zones, which 
allows maximum skin and muscle warming at each segment. The WPT were connected to a heating system, consisting of a temperature-controlled water bath and powered water pump. In the UPPER sessions, the tubing covering the lower segments of both legs was disconnected from the system.

\section{Blood pressure cuff}

Blood flow was restricted using arterial occlusion of the leg. An adjustable blood pressure cuff (Hokanson, Bellevue, WA 98005 USA) was applied around the proximal portion of the thigh, as close as possible to the groin region. For each participant, the same (right) leg was used for the blood flow intervention in each condition (WHOLE versus UPPER). The blood pressure cuff was connected by a PVC tube to a custom-made compressor able to inflate the blood cuff with a supra-systolic pressure $(140 \mathrm{mmHg})$. This was measured before the recovery period and shown to ensure full blood flow restriction.

\section{Thermal discomfort}

Thermal discomfort of the legs and whole body was recorded at 5-min intervals throughout each of the experimental trials by using a 5-point scale ('do you find this': $0=$ comfortable $;=$ slightly uncomfortable; $2=$ uncomfortable; $3=$ very comfortable; $4=$ extremely uncomfortable) (ISO 1995).

\section{Statistics}

A preliminary analysis was conducted to ensure that there were no violations to the assumption of normality of distribution. Data were analysed using 3-way repeated measures ANOVA with a Bonferroni correction. The effect of the independent variables (blood flow, heating procedure and muscle depth) was observed over the time on $T_{\text {mvl }}$ (PRE, POST-WUP and POST-REC), $T_{\mathrm{c}}, T_{\text {sk-quad }}, T_{\text {sk-gas }}$ and HR (PRE, 5WUP, 10WUP, 15WUP, 5REC, 10REC, 15REC, 20REC, 25REC and 30REC). In addition to the $p$ value, the effect size index (partial Eta squared, $\eta_{p}^{2}$ ) was included to assess the proportion of variance in $T_{\mathrm{mvl}}$ in response to the heating procedure (WHOLE versus UPPER). Effect size index is interpreted as 0.10 small effect, 0.30 medium effect and 0.50 large effect (Cohen 1988). One-way between-group ANOVA was conducted to assess the effect of the passive heating (CON; ELEC40; WPT43) on $T_{\mathrm{mvl}}$. Where significant effects were identified, post hoc multiple comparison tests with Fisher LST correction were conducted. The accepted level of significance was set a priori to $p \leq 0.05$. Data are presented as mean $\pm \mathrm{SD}$. The software SPSS I20 was used for the statistical analysis.

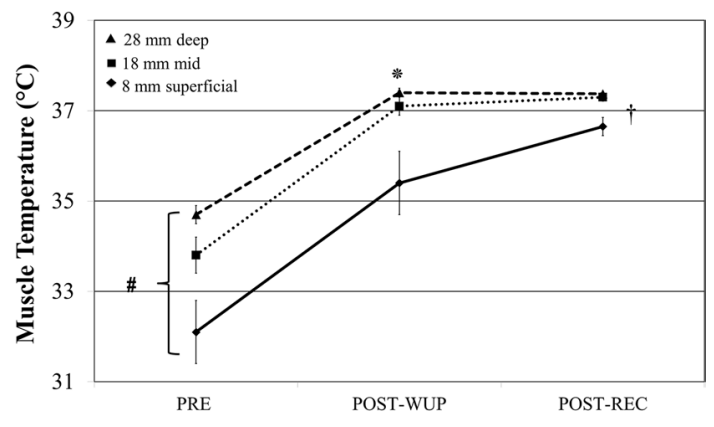

Fig. 1 Muscle temperature change from baseline (PRE) to postwarm-up (POST-WUP) and post-recovery (POST-REC) at 28, 18 and $8 \mathrm{~mm}$ depths. "Significant effect of depth on muscle temperature at both PRE and POST-WUP time points $(p<0.05)$. *Significant effect of warm-up on muscle temperature at all depths $(\mathrm{p}<0.001)$. ${ }^{\dagger} \mathrm{Sig}-$ nificant effect of passive warm-up on muscle temperature at all depth $(p<0.01)$. PRE and POST-WUP data are presented as the average of both legs. POST-REC muscle temperature is presented as the average of the perfused leg (BF) data collapsed over heating procedure WHOLE versus UPPER

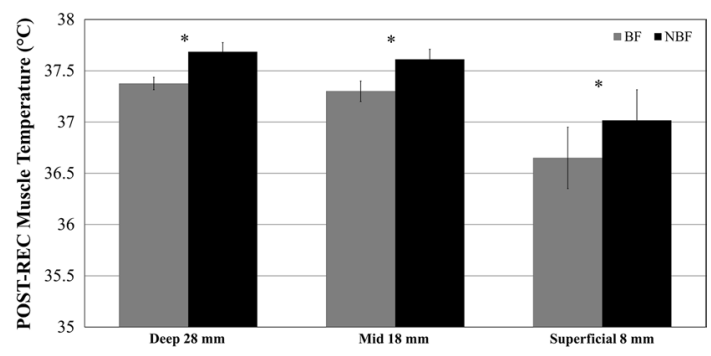

Fig. 2 Thigh muscle temperature post 30-min recovery period (POST-REC) collapsed for heating procedure (WHOLE versus UPPER) at deep $(28 \mathrm{~mm})-$, mid $(18 \mathrm{~mm})$ - and superficial- $T_{\mathrm{mvl}}$ $(8 \mathrm{~mm})$ depths. *Blood flow significantly decreased $(p<0.05)$ muscle temperature in BF (circulated leg) compared to NBF (occluded leg) at all measured depths

\section{Results}

\section{Muscle temperature}

Resting (PRE) $T_{\text {mvl }}$ at each depth was not significantly different between legs (right versus left), blood flow conditions (BF versus NBF) or heating procedure (WHOLE versus UPPER). However, PRE $T_{\mathrm{mvl}}$ did vary significantly $(p<0.05)$ across all measured depths $(28,18$ and $8 \mathrm{~mm})$ : deep- $T_{\text {mvl }}\left(34.7 \pm 0.8{ }^{\circ} \mathrm{C}\right)$ was significantly different from mid- $T_{\text {mvl }}\left(33.8 \pm 1.4{ }^{\circ} \mathrm{C}\right)$; deep- and mid- $T_{\text {mvl }}$ were significantly different from superficial- $T_{\mathrm{mvl}}\left(32.1 \pm 2.4^{\circ} \mathrm{C}\right)$. After the warm-up (POST-WUP), $T_{\text {mvl }}$ increased by $2.7 \pm 0.6$, $3.2 \pm 1.2$ and $3.3 \pm 1.3{ }^{\circ} \mathrm{C}$ above resting values for 28 , 18 and $8 \mathrm{~mm}$, respectively (Fig. 1), narrowing the temperature gradient between depths $(p<0.001)$. POST-WUP $T_{\mathrm{mvl}}$ was unaffected by blood flow conditions (BF versus NBF) and heating procedure (WHOLE versus UPPER). 
POST-REC $T_{\mathrm{mvl}}$ was not significantly different ( $p>0.05$; $\eta_{p}^{2}<0.10$ ) between heating procedure (deep- $T_{\mathrm{mvl}}$ WHOLE $37.5 \pm 0.3{ }^{\circ} \mathrm{C}$ versus deep- $T_{\text {mvl }}$ UPPER $37.5 \pm 0.2{ }^{\circ} \mathrm{C}$; mid- $T_{\text {mvl }}$ WHOLE $37.5 \pm 0.4{ }^{\circ} \mathrm{C}$ versus mid- $T_{\text {mvl }}$ UPPER $37.4 \pm 0.5^{\circ} \mathrm{C}$; superficial $-T_{\text {mvl }}$ WHOLE $36.6 \pm 1.1^{\circ} \mathrm{C}$ versus superficial- $T_{\text {mvl }}$ UPPER $37.0 \pm 0.8{ }^{\circ} \mathrm{C}$ ), and there was no interaction between heating procedure and blood flow. Therefore, $T_{\mathrm{mvl}}$ data were collapsed for heating procedure (WHOLE versus UPPER) and are presented in the two conditions BF and NBF. POST-REC $T_{\text {mvl }}$ was lower in BF versus NBF: $0.31 \pm 0.3{ }^{\circ} \mathrm{C}$ for deep- $T_{\text {mvl }} ; 0.31 \pm 0.4{ }^{\circ} \mathrm{C}$ for mid- $T_{\mathrm{mvl}}$ and $0.36 \pm 0.4{ }^{\circ} \mathrm{C}$ for superficial $T_{\mathrm{mvl}}$ (all $p<0.05$, Fig. 2).

Post-recovery period $T_{\mathrm{mvl}}$ values obtained from our previous electrical heating method (ELEC40) and control group (non-heated tracksuit bottoms; CON) were compared to POST-REC BF $\mathrm{T}_{\mathrm{mvl}}$ data achieved with the current optimised heating procedure (WPT43) (Fig. 3). In WPT43, the reduction in $T_{\text {mvl }}$ during recovery was significantly attenuated or even eliminated compared to ELEC40 and CON $(p<0.001)$. Deep- $\mathrm{T}_{\mathrm{mvl}}$ decreased by $0.12 \pm 0.3$, $1.08 \pm 0.4$ and $1.3 \pm 0.3{ }^{\circ} \mathrm{C}$ in WPT43, ELEC40 and CON condition, respectively. Mid- $T_{\text {mvl }}$ increased by $0.15 \pm 0.3{ }^{\circ} \mathrm{C}$ in WPT43, whereas it was reduced by $1.15 \pm 0.2$ and $1.44 \pm 0.2{ }^{\circ} \mathrm{C}$ in ELEC40 and CON conditions, respectively. Finally, superficial- $T_{\text {mvl }}$ increased by $1.1 \pm 1.1{ }^{\circ} \mathrm{C}$ in WPT43 condition and dropped by $0.8 \pm 0.5$ and $1.4 \pm 0.3{ }^{\circ} \mathrm{C}$ in ELEC40 and CONT conditions, respectively.

\section{Heart rate and core temperature}

There was no effect of blood flow conditions (BF versus NBF) and heating procedure (WHOLE versus UPPER) on both HR (Table 1) and $T_{\mathrm{c}}$ (Fig. 4) during the trials. In both WHOLE and UPPER conditions $T_{\mathrm{c}}$ increased significantly $(p<0.05)$ at time points 10WUP, 15WUP, 5REC, 10REC, 15REC, 20REC, 25REC and 30 REC compared to PRE and 5WUP. At time point 10REC, $T_{\mathrm{c}}$ reaches the peak and significantly decreases $(p<0.05)$ at time points 15REC, 20REC, 25REC and 30 REC compared to 10REC.

\section{Quadriceps skin temperature}

Baseline $T_{\text {sk-quad }}$ was $30.9 \pm 0.7{ }^{\circ} \mathrm{C}$ and not different between legs (right versus left), blood flow conditions (BF versus NBF) and heating procedure (WHOLE versus UPPER). During the 15 min warm-up $T_{\text {sk-quad }}$ did not increase in response to the exercise. During the recovery

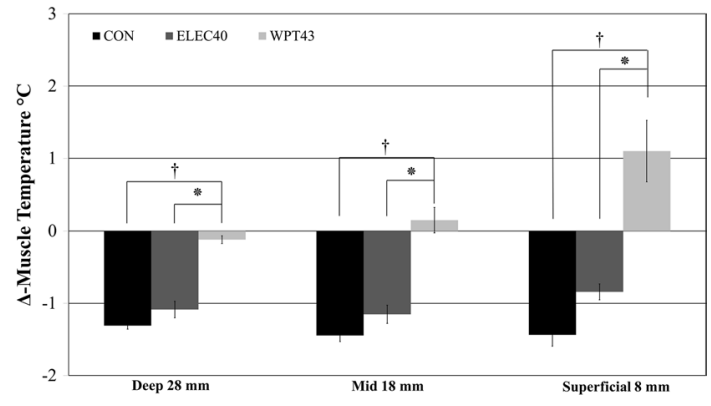

Fig. 3 Normalised muscle temperature (difference from post-warmup) recorded after $30 \mathrm{~min}$ of passive recovery following a standardised sprint cycling warm-up in CON (tracksuit bottoms), ELEC40 (electrically heated trousers at $40{ }^{\circ} \mathrm{C}$ ) and WPT43 (trousers perfused with water at $\left.43{ }^{\circ} \mathrm{C}\right)$ at deep $(28 \mathrm{~mm})$-, mid $(18 \mathrm{~mm})$ - and superficial- $T_{\mathrm{mvl}}(8 \mathrm{~mm})$ depths. ${ }^{\dagger}$ Significant effect $(p<0.001)$ of WPT43 on post-warm-up $T_{\text {mvl }}$ compared to CON. *Significant difference $(p<0.001)$ between the two heating procedures: ELEC40 and WPT43

period (REC), $T_{\text {sk-quad }}$ increased continuously in response to the passive heating, and at $30 \mathrm{~min}$ into REC, it was significantly elevated $\left(38.4 \pm 1{ }^{\circ} \mathrm{C}\right)$ above PRE $(p<0.05)$ (Table 1) but not different between conditions (BF versus NBF; WHOLE versus UPPER).

\section{Gastrocnemius skin temperature}

After the stabilisation period (PRE), $T_{\text {sk-gas }}$ was $30.4 \pm 0.9{ }^{\circ} \mathrm{C}$ with no difference between blood flow conditions (BF versus NBF) and heating procedure (WHOLE versus UPPER). During the warm-up exercise, $T_{\text {sk-gas }}$ decreased to $29.0 \pm 1.8{ }^{\circ} \mathrm{C}(p<0.05)$. During the recovery period (REC), $T_{\text {sk-gas }}$ increased continuously in response to the passive heating $(p<0.05)$. At $10 \mathrm{~min}$ into REC (10 REC), $T_{\text {sk-gas }}$ was significantly different $(p<0.001)$ between heating procedure (WHOLE versus UPPER) but not between blood flow (BF versus NBF). At the end of the recovery period (30 REC), WHOLE $T_{\text {sk-gas }}$ was $37.9 \pm 0.7{ }^{\circ} \mathrm{C}$, whereas UPPER $T_{\text {sk-gas }}$ was $34.1 \pm 1{ }^{\circ} \mathrm{C}$ (Table 1).

\section{Whole-body thermal comfort}

Thermal discomfort of the whole body was not affected by blood flow conditions (BF versus NBF) and heating procedure (WHOLE versus UPPER). Over time whole body thermal discomfort varied significantly $(p<0.01)$. After 30 min of stabilisation period (PRE), thermal comfort was 0 ("comfortable"), during the warm-up exercise increased to 1 ("slightly uncomfortable") and over the recovery period was 0.7 (between "comfortable" and "slightly uncomfortable"). 
Table 1 Quadriceps skin temperature $\left(T_{\text {sk-quad }}\right)$, Gastrocnemius skin temperature ( $\left.T_{\text {sk gas }}\right)$ for whole leg heating (WHOLE) and upper leg heating only (UPPER), core temperature $\left(T_{\mathrm{c}}\right)$ and heart rate (HR), measured after 30-mins recovery period and each 5 mins during active warm-up (WUP) and passive recovery period (REC)

\begin{tabular}{|c|c|c|c|c|c|c|c|c|c|c|}
\hline & PRE & $5 \mathrm{WUP}$ & 10WUP & 15WUP & 5REC & 10REC & 15REC & 20REC & 25REC & 30REC \\
\hline$T_{\text {sk-quad }}\left({ }^{\circ} \mathrm{C}\right)$ & $30.9 \pm 0.7$ & $30.6 \pm 0.6$ & $30.7 \pm 0.8$ & $30.6 \pm 1.1$ & $30.7 \pm 1.1$ & $32.2 \pm 1.9$ & $35.6 \pm 1.8$ & $37.3 \pm 1.5$ & $37.9 \pm 1.3$ & $38.4 \pm 1.3$ \\
\hline$T_{\text {sk-gas WHOLE }}\left({ }^{\circ} \mathrm{C}\right)$ & $30.4 \pm 1.1$ & $29.4 \pm 1.5$ & $29.2 \pm 1.7$ & $29.0 \pm 2.1$ & $29.4 \pm 2.0$ & $31.7 \pm 1.6$ & $33.7 \pm 1.9$ & $36.0 \pm 0.9$ & $36.9 \pm 0.9$ & $37.9 \pm 0.7$ \\
\hline$T_{\text {sk-gas UPPER }}\left({ }^{\circ} \mathrm{C}\right)$ & $30.4 \pm 0.8$ & $29.6 \pm 0.9$ & $29.2 \pm 1.3$ & $29.1 \pm 1.6$ & $29.5 \pm 1.8$ & $30.5 \pm 1.7$ & $31.9 \pm 1.5$ & $32.9 \pm 1.3$ & $33.4 \pm 1.2$ & $34.1 \pm 1.1$ \\
\hline$T_{\mathrm{c}}\left({ }^{\circ} \mathrm{C}\right)$ & $37.2 \pm 0.2$ & $37.2 \pm 0.2$ & $37.3 \pm 0.2$ & $37.5 \pm 0.3$ & $37.6 \pm 0.3$ & $37.8 \pm 0.3$ & $37.7 \pm 0.3$ & $37.6 \pm 0.3$ & $37.5 \pm 0.3$ & $37.4 \pm 0.1$ \\
\hline $\mathrm{HR}(\mathrm{bpm})$ & $71 \pm 10$ & $110 \pm 16$ & $153 \pm 15$ & $152 \pm 22$ & $107 \pm 12$ & $108 \pm 12$ & $106 \pm 12$ & 105 & $106 \pm 12$ & $105 \pm 12$ \\
\hline
\end{tabular}

All data presented as mean $\pm \mathrm{SD}$

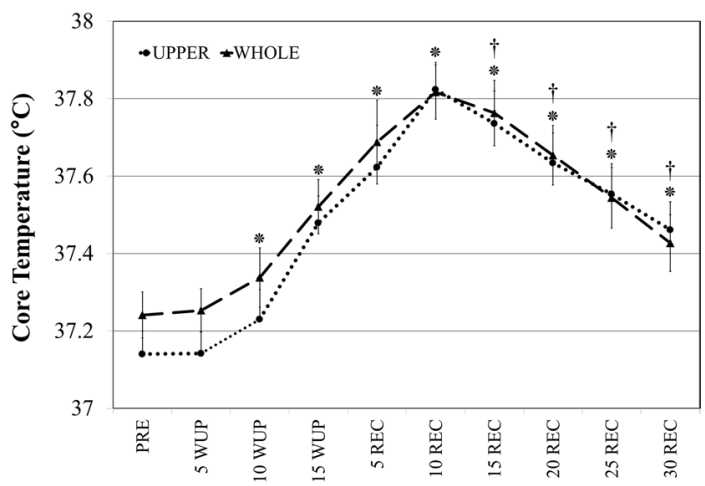

Fig. 4 Rectal temperature recorded every 5 min over the course of the trial in both WHOLE and UPPER conditions: after 30 min of stabilisation (PRE), during $15 \mathrm{~min}$ of active warm-up (5WUP; 10WUP; $15 \mathrm{WUP}$ ), and during $30 \mathrm{~min}$ of passive recovery (5REC; 10REC; 15REC; 20REC; 25REC; 30REC). *Significant increase $(p<0.05)$ in core temperature from PRE. ${ }^{\dagger}$ Significant decrease $(p<0.05)$ in core temperature from 15REC

\section{Legs thermal discomfort}

A similar trend was shown by legs' thermal discomfort; it was different $(p<0.01)$ over time, and after the stabilisation period (PRE) the score was 0 ("comfortable") and reached the value of 0.6 , between "comfortable" and "slightly uncomfortable", during the warm-up exercise. During the recovery period (REC), heating procedure (WHOLE versus UPPER) did not affect legs thermal discomfort. During REC BF, thermal discomfort increased $(p<0.05)$ to 1.6 , between "slightly uncomfortable" and "uncomfortable", whereas in NBF, it was 1.8 and not different from thermal discomfort achieved during the warm-up.

\section{Discussion}

This study demonstrates that blood perfusion of the thigh muscles contributes to the cooling of $T_{\mathrm{mvl}}$ during $30 \mathrm{~min}$ of passive recovery, following an active warm-up, even when the leg is passively heated. Conversely, heating the lower leg, in addition to the upper leg, did not affect post-recovery $T_{\mathrm{mvl}}$. Additionally, the use of an optimised heating procedure, consisting of trousers perfused with water at $43{ }^{\circ} \mathrm{C}$, instead of electric heating at $40{ }^{\circ} \mathrm{C}$, can increase mid- and superficial- $T_{\mathrm{mvl}}$ rather than just reducing the drop and considerably reduce the post-warm-up drop in deep- $T_{\mathrm{mvl}}$. Thus, we accept our hypotheses that both the central (core) blood flow and the heating temperature contribute to the reduction in post-warm up $T_{\mathrm{mvl}}$.

\section{Muscle temperature}

Confirming previous results (Saltin et al. 1968; Kenny et al. 2003), baseline $T_{\mathrm{mvl}}$ was different across all measured depths: $34.7,33.8$ and $32.1^{\circ} \mathrm{C}$, for deep-, mid- and superficial- $T_{\mathrm{mvl}}$, respectively. This is in line with the work of Saltin et al. (1968) who found that at rest deep- $T_{\mathrm{mvl}}$ is around 33-34 ${ }^{\circ} \mathrm{C}$. In the current study, warm-up exercise increased $T_{\text {mvl }}$ by $2.7,3.2$ and $3.3{ }^{\circ} \mathrm{C}$ for deep-, mid- and superficialdepths, respectively, reducing the temperature gradient across the three depths. Additionally, after $30 \mathrm{~min}$ of passive recovery, $T_{\mathrm{mvl}}$ of the perfused leg was lower compared to $T_{\text {mvl }}$ of the occluded leg at all measured depths $\left(\sim 0.3{ }^{\circ} \mathrm{C}\right.$ for deep- and mid- $T_{\mathrm{mvl}} ; \sim 0.4{ }^{\circ} \mathrm{C}$ for superficial- $\left.T_{\mathrm{mvl}}\right)$. In the perfused leg, the optimised external temperature resulted in a small decrease in deep- $T_{\mathrm{mvl}}$ and in an increase in mid- and superficial- $T_{\text {mvl }}\left(0.15\right.$ and $1.1{ }^{\circ} \mathrm{C}$, respectively) compared to post-warm-up values.

\section{The role of external temperature on post-warm-up muscle temperature}

A central aim of this study was to examine the role of passive heating temperature on the decline in post-warm-up $T_{\mathrm{mvl}}$ and whether a relatively small increase in heating temperature $\left(40-43{ }^{\circ} \mathrm{C}\right)$ would provide a measurable benefit. The heating temperature of the previous trousers was set to a maximum of $40{ }^{\circ} \mathrm{C}$. Since the trousers were electrically heated, it was not possible to control the local heating temperature precisely and further increases in the heating 
element's temperature could have caused local overheating and skin burns. During piloting for this study, we observed that an external temperature of $40{ }^{\circ} \mathrm{C}$ resulted in a mean leg $T_{\mathrm{sk}}$ of $\sim 37{ }^{\circ} \mathrm{C}$, which was not enough to maintain $T_{\mathrm{mvl}}$ achieved with the active warm-up (Faulkner et al. 2013a, b). The use of circulated liquid heating allowed the safe application of an optimised, higher heating temperature $\left(43{ }^{\circ} \mathrm{C}\right)$ which resulted in a higher quadriceps $T_{\text {sk }}\left(\sim 38^{\circ} \mathrm{C}\right.$; Table 1), thus minimising the temperature gradient between $T_{\mathrm{sk}}$ and $T_{\mathrm{mvl}}$. The optimisation of the heating procedure lead to a substantial reduction of the deep- $T_{\mathrm{mvl}}$ drop compared to our previous studies (Faulkner et al. 2013a, b), where we reported $\mathrm{a} \sim 1{ }^{\circ} \mathrm{C}$ decline, and to increases rather than decreases in mid and superficial- $T_{\mathrm{mvl}}$. The present data indicate that microclimate temperature is a major contributing factor in post-warm-up $T_{\mathrm{mvl}}$ decline and that even relatively small changes in heating temperature have an impact. Furthermore, the greater increase in superficial- $T_{\mathrm{mvl}}$ indicates, as to be expected, that the superficial tissues are most affected by microclimate and ambient temperature, which may be the most important for cycling sprint performance (Faulkner et al. 2013b).

\section{The contribution of blood flow on post-warm-up muscle temperature}

The higher $T_{\mathrm{mvl}}$ in the occluded condition confirms the cooling effect of blood flow. This could be by arterial blood from the core, venous blood from the lower leg, or both. The fact that rectal temperature remains higher than muscle temperature throughout the recovery period would suggest that arterial blood could not be responsible. However, rectal temperature is often higher $\left(\sim 0.3{ }^{\circ} \mathrm{C}\right)$ than arterial blood temperature (Taylor et al. 2014), and oesophageal temperature, better representing arterial temperature, decreases faster than rectal temperature post-exercise (Gagnon et al. 2010). Therefore, it is likely that arterial blood temperature entering the thigh was lower than muscle temperature, during, at least, part of the recovery period; this would support a role of arterial blood flow on post-warm-up muscle temperature reduction, as observed in the present study. However, since in this study arterial blood temperature was not measured, the idea that it may have been lower than muscle temperature remains an assumption.

The temperature difference in $T_{\mathrm{mvl}}$ between perfused and occluded leg was relatively small $\left(\sim 0.3\right.$ to $\sim 0.4{ }^{\circ} \mathrm{C}$ for different depths) which could be due to the high heating temperature used in this study. In fact, it is possible that the optimised passive heating could also reduce the drop rate in central and peripheral blood temperature post-warmup, reducing the temperature gradient between blood and muscle and therefore narrowing the temperature difference between the perfused and occluded leg. Nevertheless, the effect of blood flow on $T_{\mathrm{mvl}}$ is still evident, though it would likely have been more pronounced with the electric heating system at $40{ }^{\circ} \mathrm{C}$ (Faulkner et al. 2013a, b).

We hypothesised that peripheral blood, together with central blood flow, could contribute to the post-warm-up $T_{\mathrm{mvl}}$ decline. To verify our hypothesis, post-recovery $T_{\mathrm{mvl}}$ was observed in two different conditions: WHOLE and UPPER. However, while we expected a lower thigh $T_{\mathrm{mvl}}$ in the UPPER condition compared to WHOLE condition, thigh muscle temperature was not different between heating interventions. It is possible that the high blood temperature (resulting from the higher heating temperature used) flowing from the thigh to the lower leg, increased the temperature of the lower leg tissue in the UPPER condition. In support of this, skin temperature of the lower leg and foot showed a significant increase of $4{ }^{\circ} \mathrm{C}$ during the recovery period even if heat was not directly applied, whereas in the whole leg heated condition (WHOLE), it increased by $7{ }^{\circ} \mathrm{C}$. Therefore, these results indicate that, when using an optimised heating method, heating the calf in addition to the thigh does not further increase quadriceps muscle temperature, compared to heating the thigh only. However, for sports where calf muscles are relevant, lower leg heating may still provide a performance benefit due to increased local $T_{\mathrm{m}}$.

Blood flow restriction coupled with the use of passive heating resulted in a $T_{\text {mvl }}$ increase at all depths. In this study, blood flow occlusion was used as means to understand the mechanistic effect of circulating blood flow on post-warm-up $T_{\mathrm{mvl}}$ decline, rather than a proposed tool to maintain $T_{\mathrm{mvl}}$ during a period of inactivity. In fact, $30 \mathrm{~min}$ of blood flow occlusion is expected to have a detrimental effect on performance, as the restriction of circulation would not allow the wash-out of metabolites accumulated in the leg during exercise, causing muscle discomfort and peripheral fatigue (Bigland-Ritchie et al. 1986; Gandevia et al. 1996; Amann et al. 2013).

As the study of blood flow precluded doing a performance test, it needs to be considered whether the observed improved maintenance of $T_{\mathrm{m}}$ would result a in further sprint-power performance enhancement above those observed in Faulkner et al.'s (2013a, b) muscle heating studies. Optimal $T_{\mathrm{m}}$ is thought to be $\sim 39{ }^{\circ} \mathrm{C}$, with an upper threshold of $43{ }^{\circ} \mathrm{C}$, above which there are impairments to muscle function (Åstrand and Rodahl 1986; McRae and Esrick 1993). Although the relationship between improved sprint performance and increased $T_{\mathrm{m}}$ may not follow complete linearity, Zochowski et al. (2007) and West et al. (2013) showed that shorter recovery periods (down to 10 and $20 \mathrm{~min}$ from $45 \mathrm{~min}$ ) between warm-up and race, with concomitant smaller drops in $T_{\mathrm{m}}$, did result in a greater improvement in the following sprint performance. The latter strongly indicate that the smaller the post-warm-up $T_{\mathrm{m}}$ drop, the better the subsequent sprint/power performance, 
thus supporting that the optimised heating method used here has a strong potential of further performance enhancement.

\section{Conclusion}

This study has demonstrated that to maintain post-warmup muscle temperature, an increase of the leg heating temperature from 40 to $43{ }^{\circ} \mathrm{C}$ is sufficient to virtually abolish the deep thigh muscle temperature drop. Additionally, a $3{ }^{\circ} \mathrm{C}$ higher external temperature can even increase superficial muscle temperatures during a 30-min recovery period. Further, it was observed that blood perfusing the thigh during the period of inactivity following an active warm-up is one of the factors responsible for the earlier observed reductions in thigh muscle temperature. Heating the lower leg in addition to the upper leg however did not further improve thigh muscle temperature. Nevertheless, in an applied sports performance setting, additional performance benefits from lower leg heating on the lower leg muscles may be evident. Given the cooling effect of blood flow observed, and according to the local heat balance, lower leg heating could also improve upper leg $T_{\mathrm{mvl}}$ in less optimised heating systems as used in earlier studies from our laboratory.

Given that the water-perfused heating system in its current form may not be practical for use in the field, further product development needs to be considered by the sporting goods industry, now that the present study has demonstrated that optimisation of the heating system can provide further gains in muscle temperature maintenance.

\section{Compliance with ethical standards}

\section{Conflict of interest None.}

Open Access This article is distributed under the terms of the Creative Commons Attribution 4.0 International License (http://creativecommons.org/licenses/by/4.0/), which permits unrestricted use, distribution, and reproduction in any medium, provided you give appropriate credit to the original author(s) and the source, provide a link to the Creative Commons license, and indicate if changes were made.

\section{References}

Amann M, Venturelli M, Ives SJ, McDaniel J, Layec G, Rossman MJ, McDaniel J, Layec G, Rossman MJ, Richardson RS (2013) Peripheral fatigue limits endurance exercise via a sensory feedback-mediated reduction in spinal motoneuronal output. J Appl Physiol 115(3):355-364

Asmussen E, Boje O (1945) Body temperature and capacity for work. Acta Physiol Scand 10(1):1-22
Åstrand PO, Rodahl K (1986) Textbook of work physiology. Physiological bases of exercise, 3rd edn. McGraw-Hill Book Co, New York

Bergh U, Ekblom B (1979) Influence of muscle temperature on maximal muscle strength and power output in human skeletal muscles. Acta Physiol Scand 107(1):33-37

Bigland-Ritchie BR, Dawson NJ, Johansson RS, Lippold OC (1986) Reflex origin for the slowing of motoneurone firing rates in fatigue of human voluntary contractions. J Physiol 379:451-459

Bishop D (2003a) Warm up I: potential mechanisms and the effects of passive warm up on exercise performance. Sports Med 33(6):439-454

Bishop D (2003b) Warm up II: performance changes following active warm up and how to structure the warm up. Sports Med 33(7):483-498

Bishop D, Bonetti D, Dawson B (2001) The effect of three different warm up intensities on sprint kayak performance in trained athletes. Med Sci Sports Exerc 33(6):1026-1032

Cohen J (1988) Statistical power analysis for the behavioral sciences. Erlbaum, Hillsdale

Dawson B, Goodman C, Lawrence S et al (1997) Muscle phosphocreatine repletion following single and repeated short sprint efforts. Scand J Med Sci Sports 7(4):206-213

Ducharme MB, Tikuisis P (1994) Role of blood as heat source or sink in human limbs during local cooling and heating. J Appl Physiol 76(5):2084-2094

Faulkner SH, Ferguson RA, Gerrett N, Hupperets M, Hodder SG, Havenith G (2013a) Reducing muscle temperature drop after warm-up improves sprint cycling performance. Med Sci Sport Exerc 45(2):359-365

Faulkner SH, Ferguson RA, Hodder SG, Havenith G (2013b) External muscle heating during warm-up does not provide added performance benefit above external heating in the recovery period alone. Eur J Appl Physiol 113(11):2713-2721

Gagnon D, Lemire BB, Jay O, Kenny GP (2010) Aural canal, esophageal, and rectal temperatures during exertional heat stress and the subsequent recovery period. J Athl Train 45(2):157-163

Gandevia SC, Allen GM, Butler JE, Taylor JL (1996) Supraspinal factors in human muscle fatigue: evidence for suboptimal output from the motor cortex. J Physiol 490:529-536

Hajoglou A, Foster C, De Koning JJ, Lucia A, Kernozek TW, Porcari JP (2005) Effect of warm-up on cycle time trial performance. Med Sci Sport Exerc 37(9):1608-1614

Havenith G (2001) Individualized model of human thermoregulation for the simulation of heat stress response. J Appl Physiol 90(5):1943-1954

ISO 10051 FS (1995) Ergonomics of the thermal environment-assessment of the influence of the thermal environment using subjective judgement scales. International Organization for Standardization, Geneva

Kenny GP, Reardon FD, Zaleski W, Reardon ML, Haman F, Ducharme MB (2003) Muscle temperature transients before, during, and after exercise measured using an intramuscular multisensor probe. J Appl Physiol 94(6):2350-2357

Kilduff LP, West DJ, Williams N, Cook CJ (2013) The influence of passive heat maintenance on lower body power output and repeated sprint performance in professional rugby league players. J Sci Med Sport 16(5):482-486

Malareki I (1954) Investigation of physiological justification of socalled 'warming-up'. Acta Physiol Pol 5(1):543-546

McRae DA, Esrick MA (1993) Changes in electrical impedance of skeletal muscle measured during hyperthermia. Int J Hyperthermia 9(2):247-261

Mohr M, Krustrup P, Nybo L, Nielsen JJ, Bangsbo J (2004) Muscle temperature and sprint performance during soccer 
matches-beneficial effect of re-warm-up at half-time. Scand J Med Sci Sports 14(3):156-162

Saltin B, Gagge AP, Stolwijk JA (1968) Muscle temperature during submaximal exercise in man. J Appl Physiol 25(6):679-688

Sargeant AJ (1987) Effect of muscle temperature on leg extension force and short-term power output in humans. Eur J Appl Physiol Occup Physiol 56(6):693-698

Taylor NAS, Tipton MJ, Kenny GP (2014) Considerations for the measurement of core, skin and mean body temperatures. J Therm Biol 46:72-101
West DJ, Dietzig BM, Bracken RM, Cunningham DJ, Crewther BT, Cook CJ et al (2013) Influence of post-warm-up recovery time on swim performance in international swimmers. J Sci Med Sport 16(2):172-176

Zochowski T, Johnson E, Sleivert GG (2007) Effects of varying postwarm-up recovery time on $200-\mathrm{m}$ time-trial swim performance. Int J Sports Physiol Perform 2:201-211 